\title{
RESEARCH AND DEVELOPMENT OF RESONANT ELECTROMAGNETIC VIBRATION SCREEN FOR INTENSIVE VIBRATION TECHNOLOGIES
}

\author{
Almukhan Nuraliyev ${ }^{1}$, Mukhtorkhon Ibadullayev ${ }^{2}$ \\ ${ }^{1}$ Doctor of technical sciences, senior lecturer of "Electrical Engineering" department, Tashkent State Technical University. \\ ${ }^{2}$ Doctor of technical sciences, professor of "Electrical Engineering" department, Tashkent State Technical University.
}

\begin{abstract}
Annotation. In the article the development and use of controlled electromagnetic vibration drives used in the field of intensive vibration technologies are considered. Method is proposed for adjustment of amplitude and frequency of vibration of working element of vibrating screen, which improves efficiency of cutting, its dynamic and energy indices. By solving the differential equations of the system, its main characteristics, which can be used in the calculation and design of electromagnets, are determined. For experimental research, a vibrator was developed with a drive from a two-stroke electromagnetic exciter. Based on the results of the studies, it was established that the adjustment of the near-resonance mode when the load changes can always be provided by changing the frequency of the thyristor converter voltage pulses. Optimal combinations of amplitudes and oscillation frequencies of the developed vibration sieve are determined.
\end{abstract}

\section{Introduction}

A number of modern technological processes and formation of products (sifting, filtering, sorting, hashing, cleaning, etc.) can be considerably intensified due to use of vibration effects with the frequency range of $20-50 \mathrm{~Hz}$ and with amplitude of fluctuations up to $5 \mathrm{~mm}$. For these purposes, the promising use of electromagnetic exciters of mechanical vibrations, which have sufficient structural simplicity, reliability and provide for the creation of vibration effects with the indicated frequency range and vibration amplitudes. The design and operational features of these machines make it possible to successfully use them in modern automated technological processes $[4,5$, $6,7,10,11,12]$.

Compared to other types of vibration drivers of mechanical vibrations, electromagnetic ones are characterized by durability in operation, ease of maintenance, low operating costs and wide automation capabilities.

A distinctive feature of the operation of these machines is the high vibration intensity created by electromagnetic vibration drivers, due to the high frequency and relatively small amplitudes $[4,6,7,10,11,12]$.

For experimental research, a laboratory sample of a vibrating screen driven by a two-stroke electromagnetic exciter was developed.
The schematic diagram of the developed vibration screen is given in Fig. 1. It consists of two-stroke electromagnetic vibration exciter with two $\mathrm{W}$-shaped electromagnets 1 , with windings 2 and armatures 3 connected through elastic elements 4 to fixed part of EMVE (electromagnet cores). Frame of screen web 5 is connected through spring elements 4 to stands of vibrating screen bed.

To control oscillation frequency, semiconductor frequency converter of parallel type is used. The frequency of mechanical oscillations is controlled by a control pulse generator (multivibrator or flip-flop).

EMVE windings are connected to DC source so that thyristors VS1 and VS2 are connected to each winding arm. Diodes VD1 and VD2 serve to eliminate overvoltage on thyristors during switching. An experimental study of the vibration sieve included in this scheme confirmed the possibility of using it in practice with a frequency control range of up to $50 \mathrm{~Hz}$.

For reliable and efficient operation of the vibration screen, it is necessary to determine the main characteristics (oscillation amplitudes, electromagnetic forces, magnetic currents, currents in windings, etc.) that serve in calculations and design. 


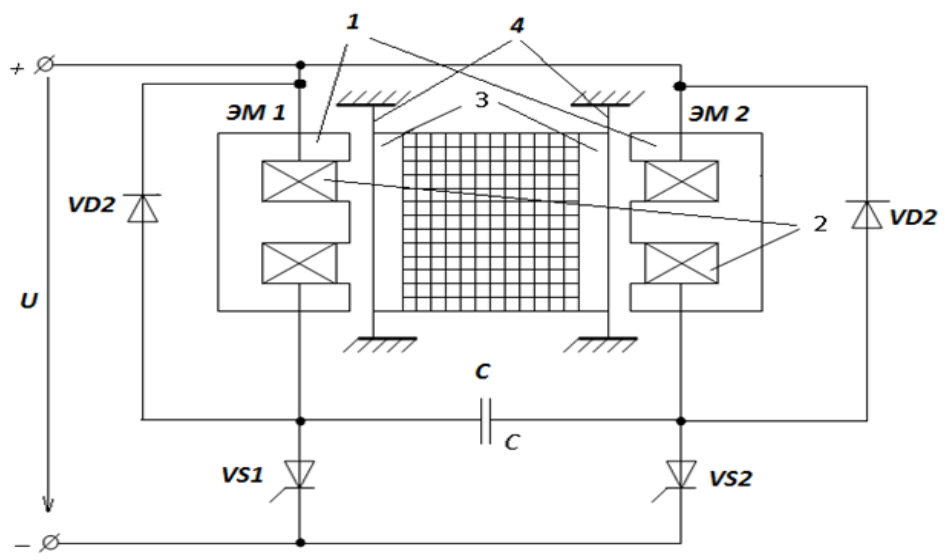

Fig.1.Schematic diagram of electromagnetic vibration screen

The analyzed system consists of two subsystems mechanical oscillation and EMVE oscillation exciter with frequency converter. The electromagnetic vibrator speaker is then described by the following equations:

$$
\left\{\begin{array}{c}
\frac{d \psi_{1}}{d t}+\frac{R_{1}}{L_{1}}=u_{1}(t) \\
\frac{d \psi_{2}}{d t}+\frac{R_{2}}{L_{2}}=u_{2}(t) \\
M \frac{d x^{2}}{d t^{2}}+k \frac{d x}{d t}+c x=F\left(x, i_{1}, i_{2}\right)
\end{array}\right.
$$

where $u_{1}(t), u_{2}(t)$ - voltages at EMVE windings,

$i_{1}, i_{2}$ - currents in windings,

$L_{1}, L_{2}$ - inductance of electromagnetic system,

$R_{1}, R_{2}$ - active winding resistances,

$x$ - movement of movable part with armature,

$k$ - friction coefficient,

$c$ - stiffness of elastic elements,

$M$ - armature weight with attached load,

$F\left(x, i_{1}, i_{2}\right)$ - electromagnetic force, which is a function of currents $i_{1}, i_{2}$ and movement $x$.

The applied voltages on the electromagnets windings are rectangular time functions shifted by an angle $\pi$ and are described by expressions

$$
\left\{\begin{array}{l}
u_{1}(t)=u_{0}\left(1-m_{0} \operatorname{sign} \omega_{B} t\right) \\
u_{2}(t)=u_{0}\left(1+m_{0} \operatorname{sign} \omega_{B} t\right)
\end{array}\right.
$$

where $u_{0}$ - constant component of voltage, $\omega_{B}$ - frequency of voltage change set by sound generator, $m_{0}-$ voltage variation factor.

The inductance of the electromagnetic system depends on the movement and can be determined from the ratios:

$$
\begin{gathered}
L_{1}=\frac{L_{0}}{1-\beta \frac{x}{x_{0}}} \\
L_{2}=\frac{L_{0}}{1+\beta \frac{x}{x_{0}}}
\end{gathered}
$$

where $L_{0}$ - inductance in armature middle position, $x_{0} \quad-$ middle clearance between armature and electromagnet core,

$\beta$ - inductance modulation coefficient.

For an unsaturated magnetic system with an average gap $x_{0}$ flux coupling can be expressed:

$$
\begin{gathered}
\psi_{1}=L_{1} i_{1} \\
\psi_{2}=L_{2} i_{2}
\end{gathered}
$$

Under these conditions, the traction force acting on the oscillating system will be:

$$
F\left(x, i_{1}, i_{2}\right)=\frac{\psi_{1}^{2}}{2 L_{1}^{2}} \frac{d L_{1}}{d x}-\frac{\psi_{2}^{2}}{2 L_{2}^{2}} \frac{d L_{2}}{d x}
$$

Decomposing (2) in the Fourier series and introducing dimensionless values into (1):

$$
\begin{array}{ll}
y=\frac{x}{x_{0}}, & F_{1}=\frac{\pi \psi_{1} \omega}{4 U_{0} m_{0}}, \\
\tau=\omega t, & F_{2}=\frac{\pi \psi_{2} \omega}{4 U_{0} m_{0}}
\end{array}
$$

we will have:

$$
\left\{\begin{array}{l}
\frac{d F_{1}}{d \tau}+\mu(1-\beta y) F_{1}=\alpha+\sum_{n} \frac{1}{n} \sin n \tau \\
\frac{d F_{2}}{d \tau}+\mu(1+\beta y) F_{2}=\alpha+\sum_{n} \frac{1}{n} \sin n \tau \\
\frac{d^{2} F_{1}}{d \tau^{2}}+\delta \frac{d y}{d t}+\omega_{0}^{2} y=F_{0}=\left(F_{1}^{2}-F_{2}^{2}\right)
\end{array}\right.
$$

Here are the following designations are accepted:

$$
\delta=\frac{\kappa}{m \omega}, \quad \omega_{0}^{2}=\frac{c}{m \omega^{2}}, \quad \mu=\frac{R_{1}}{L_{0 \omega}}=\frac{R_{2}}{L_{0 \omega}},
$$




$$
F_{0}=\frac{16 U_{0}^{2} m_{0}^{2} \beta}{2 L_{0} \pi^{2} x_{0}^{2} M \omega^{2}}, \quad a=\frac{\pi}{4 m_{0}}, \quad n=1,3,5 \ldots \ldots
$$

Considering that the value $\mu$ included in (7) is sufficiently small and can be taken as a small parameter, and the value $\alpha$ is not much different from $\mu$, periodic solutions of equation (7) can be found in series by the powers of the small parameter.
With the equality of the constant component flows in the windings of the electromagnet, the generating solutions of equations (7) are obtained in the form:

$$
\left\{\begin{array}{c}
F_{1}=\lambda_{1}-\cos \tau \\
F_{2}=\lambda_{2}+\cos \tau \\
\lambda_{1}=\lambda_{2}=\lambda=\frac{\alpha L M}{y=-\frac{1-2 \beta K_{0} K_{1} \cos \varphi_{1}}{\mu\left(1-2 \beta F_{0} K_{1} \cos \varphi_{1}\right)} \cos \left(\tau-\cos \varphi_{1}\right)}
\end{array}\right.
$$

Where:

$$
\kappa_{1}=\frac{1}{\sqrt{\left(\omega_{0}^{2}-1\right)^{2}+\delta^{2}}}, \quad \varphi_{1}=\operatorname{arctg} \frac{\delta}{1-\omega_{0}^{2}}
$$

Then for the traction force applied to the working body (sieve plate), we have:

$$
F\left(x, i_{1}, i_{2}\right)=-\frac{4 \alpha F_{0}}{\mu\left(1-2 \beta F_{0} K_{1} \cos \varphi_{1}\right)} \cos \tau
$$

Using the ratios (9) and (11), approximate expressions can be found for the current and power consumed by each electromagnet.

$$
i_{1}=\frac{4 U_{0} m_{0}}{\pi \omega L_{0}}(\lambda-\cos \tau)\left[1+4 \lambda K_{1} \cos \left(\tau-\varphi_{1}\right)\right]
$$

Under these conditions, the average power consumed by each electromagnet will be equal to

$$
P_{1}=\frac{1}{T} \int_{0}^{T} i_{1} d \psi_{1}=\frac{32 m_{0}^{2} U_{0}^{2} \lambda^{2} \beta K_{1} F_{0} \sin \varphi_{1}}{L_{0} \pi^{2} \omega^{2}}
$$

where,

$$
\lambda=\frac{\alpha}{\mathrm{M}}:\left(1-2 \beta F_{0} K_{1} \cos \varphi_{1}\right)
$$

Analysis of the obtained ratios for the electromagnetic vibration sieve in the two-stroke mode when powered by the thyristor converter shows that the processes qualitatively coincide with those occurring during the operation of the two-stroke EMVE connected by a differential circuit with constant magnetization and supply from a source of sinusoidal voltage. However, in this case, adjustment to the near resonance mode when the load (mass of drilling fluid) changes can always be provided by changing the frequency of the thyristor converter voltage pulses.

For horizontal vibration screens with rectilinear fluctuations (in our case for EMVE) perhaps also use of the following empirical formulas for determination of parameters of fluctuations $=\frac{4+140 \lambda}{1000}, \quad n=\frac{5(1+12,5 \lambda)}{a}$, where the n-frequency of fluctuations of a sieve plate in a quantity/mines, the sieve opening $\lambda$-size in $\mathrm{mm}, a$ - amplitude of fluctuations in $\mathrm{mm}$, a tilt angle of a sieve plate $\varphi$-at the same time can be $15-20^{\circ}$.

Checking by these formulas of the curve optimal combinations of amplitudes and frequencies of oscillations showed their permissible coincidence.

In Fig. 2. shows the curve of optimal combinations of amplitudes and frequencies of oscillations experimentally obtained for the developed vibration screen.

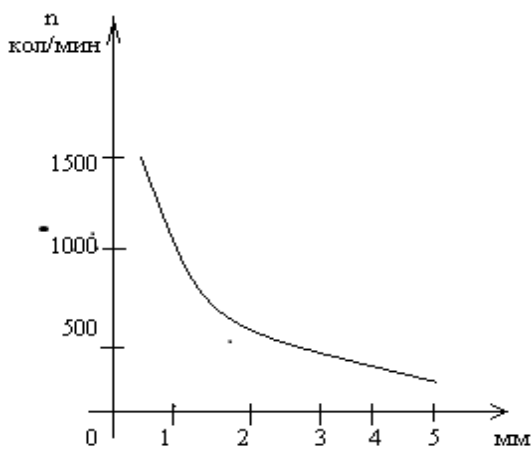

Fig.2. Curve of optimal combinations of amplitudes and frequencies of oscillations

The present study solves some of the issues for the screening of non-heavy drilling fluids using three-stage cleaning $[4,7,10,11]$.

The developed control system provides the required frequency range within $10-30 \mathrm{~Hz}$. At the same time, experimental studies showed that $22-28 \mathrm{~Hz}$ is optimal. Amplitude of oscillations can be adjusted within 2-5 mm. 
As the size of the holes of the sieve web decreases below $0.3 \mathrm{~mm}$, the vibration amplitude should decrease and the oscillation frequency should increase.

At the same time, on the sections of the screen, natural oscillations are excited, which are superimposed on the forced ones, due to which self-cleaning of the screen holes occurs even when screening difficult solutions.

\section{Conclusions}

1. The results of the studies show that tuning for resonance when changing the parameters of the mechanical subsystem can always be carried out by changing the pulse frequency of the thyristor converter.

2. The designed drive of the vibration screen for cleaning drilling fluids provides controllability of vibration parameters when changing operation modes without stopping the process.

3 . The developed control system provides the required frequency range within 15-30 $\mathrm{Hz}$. The optimum frequency is 22-28 Hz. Amplitude of oscillations can be adjusted within 2-5 $\mathrm{mm}$. The vibration amplitude shall decrease and the oscillation frequency shall increase as the sieve plate holes decrease below $0.3 \mathrm{~mm}$.

\section{References}

1. M.M. Ilyin. Theory of oscillations: Textbook for universities edited by K.S. Kolesnikov, 2nd edition. Publishing house of MSTU named after N.E.Bauman, 2003-p. 272

2. L.A. Bessonov. Nonlinear electrical circuits, 5th edition, Moscow, Higher School 2015, p.375.

3. N.N. Bogolyubov, Yu.A. Mitropolskiy. Asymptotic methods in the theory of nonlinear oscillations. Pub. 8th Moscow Science 2008, p.504.

4. M.I.Ibadullayev, A.K.Nuraliyev, A.Zh.Yesenbekov, A.I. Nazarov, Resonant electromagnetic vibration exciter with circuit reetrancy. MEI Reporter №1 2020, p.57-60. Moscow.

5. A.A.Chesnokov. To the theory and calculation of electromagnetic oscillations. Electricity. 1961 №12, p.37-40.

6. Z.I. Ismailov, I.A.Khalilov, A.Z.Ismailov. Study of two-stroke vibration exciter in forced mode. Uzbek Journal of Computer Science and Energy. Tashkent, 2017 №1, p.58-62.

7. A.I. Nazarov, M.I. Ibadullayev, M.M. Tillyakhodjayev. Structural diagram of electromagnetic vibration exciter with amplitude-frequency control. Journal of Energy and Resource Saving Problems, Tashkent, 2016 №3-4, p.55-59.

8. Hoshimov, F.A., Bakhadirov, I.I., Erejepov, M., Djumamuratov, B. (2019) Development of method for normalizing electricity consumption E3S Web Conf 139 doi:10.1051/e3sconf/201913901074

9. S.A. Shegolev. The method of the small parameter A. Puancare in the theory of nonlinear oscillations. Teaching Manual. Odessa OSU, 2015.

10. M.M. Tillyakhodjayev, M.I. Ibadullayev, A.K. Nuraliyev. Synthesis of two-stroke electromagnetic vibration exciter made according to differential scheme. Tashkent STU Reporter, №4, 2003, p.57-60

11. S.H. Chowdhury, Tilliakhojaev M., Md.S.Ullah. An Analysis on Electro-Magnetic Vibro-Exciter Fed by Non-linear Power, controlled by Velocity transducer. Journal of Electrical Engineering, the Jnstitution of Engineers, Bangladesh. Vol. EE24, №1, 1996.

12. NN.K. Nuraliyev, M.M. Tillyakhodjayev, M.I. Ibadullayev, A.Nishanbayev. Energetic readings of electromagnet vibration exciters and ways of increasing them //VCIS-2006 International conference. Intellectual systems for industrial automation - Tashkent 2006, November 21-22, p.156-158 